\title{
CLUSTERS AND SEEDS IN ACYCLIC CLUSTER ALGEBRAS
}

\author{
ASLAK BAKKE BUAN, BETHANY R. MARSH, IDUN REITEN, \\ AND GORDANA TODOROV, \\ WITH AN APPENDIX COAUTHORED IN ADDITION BY P. CALDERO AND B. KELLER
}

(Communicated by Martin Lorenz)

\begin{abstract}
Cluster algebras are commutative algebras that were introduced by Fomin and Zelevinsky in order to model the dual canonical basis of a quantum group and total positivity in algebraic groups. Cluster categories were introduced as a representation-theoretic model for cluster algebras. In this article we use this representation-theoretic approach to prove a conjecture of Fomin and Zelevinsky, that for cluster algebras with no coefficients associated to quivers with no oriented cycles, a seed is determined by its cluster. We also obtain an interpretation of the monomial in the denominator of a non-polynomial cluster variable in terms of the composition factors of an indecomposable exceptional module over an associated hereditary algebra.
\end{abstract}

\section{INTRODUCTION}

Cluster algebras were introduced by Fomin-Zelevinsky in [FZ1], and have become increasingly important in many different parts of algebra and beyond. We only consider the case involving only skew-symmetric matrices and "no coefficients". Essential ingredients in the definition (in this case) are certain $n$-element subsets $\underline{u}=\left\{u_{1}, \ldots, u_{n}\right\}$ of the function field $\mathbb{Q}\left(x_{1}, \ldots, x_{n}\right)$ called clusters, and pairs $\left(\underline{u}, Q^{\prime}\right)$ called seeds, where $\underline{u}$ is a cluster and $Q^{\prime}$ a finite quiver with no loops or 2-cycles (see Section 1 for definitions). Here $Q^{\prime}$ corresponds to a skew-symmetric integer matrix.

In this paper we only consider the acyclic case, i.e. cluster algebras for which there exists a seed $\left(\underline{u}, Q^{\prime}\right)$, where $Q^{\prime}$ is a quiver without oriented cycles. When we later refer to the acyclic case, the additional restrictions mentioned above are also assumed.

The aim of this paper is to prove that under our assumptions a seed $\left(\underline{u}, Q^{\prime}\right)$ is determined by the cluster $\underline{u}$, as conjectured by Fomin-Zelevinsky, who proved it for cluster algebras of finite type [FZ2].

Our method uses the theory of cluster categories and cluster-tilted algebras from [BMRRT], [BMR1] and [BMR2]. These were introduced to model some of the ingredients in the theory of cluster algebras in a module theoretic/categorical way, inspired by [MRZ], with the hope of obtaining feedback to the theory of cluster algebras. The proof of our result gives another illustration of this principle.

Received by the editors December 1, 2005 and, in revised form, June 4, 2006.

2000 Mathematics Subject Classification. Primary 16G20, 16G70. 
Suppose that $x$ is a non-polynomial cluster variable written in reduced form. Then the denominator is known to be a monomial [FZ1]. An essential ingredient in our proof is to introduce the notion of positivity condition, which gives an easy criterion for an expression to be in reduced form. Along the way we obtain an interpretation of the monomial in the denominator in terms of the composition factors of an indecomposable exceptional module over an associated hereditary algebra, namely the path algebra of $Q$, where $Q$ is a quiver with no oriented cycles appearing in a seed. Each indecomposable exceptional module corresponds to the denominator of a cluster variable this way. This extends previous results for finite type. The proof is also different from the earlier proofs in the case of finite type. For finite type, we also obtain a new proof of the existence of a bijection between cluster variables and indecomposable objects in the corresponding cluster category inducing a bijection between clusters and tilting objects, as first proved in [BMRRT]. In [BMRRT] it was also conjectured that beyond finite type, there should be a bijection between the exceptional objects of the cluster category and the cluster variables.

That a seed is determined by its cluster in the acyclic case is also easily deduced from the proof of Theorem 4 in [CK2]. (The result is stated in [CK2] as a consequence of Theorem 4, which depends on this paper.) While both approaches heavily use results from [BMRRT] and [BMR2], there is an essential difference. We define a surjective map $\alpha$ from cluster variables to the indecomposable exceptional objects in the cluster category, using our elementary notion of positivity. In [CK2] a surjective map $\beta$ is defined in the opposite direction by extending a beautiful formula in $[\mathrm{CC}]$ beyond finite type. Our approach gives the extra result on denominators. However, combining the two surjectivity results gives directly stronger results; see the Appendix.

Throughout this paper, $k$ denotes an algebraically closed field.

\section{BACKGROUND}

In this section we give some relevant background material for our work.

1.1. Cluster algebras. We recall the main ingredients in the definition of cluster algebras from [FZ1], without dealing with the most general setting. We denote by $\mathbb{Q}\left(x_{1}, \ldots, x_{n}\right)$ the function field in $n$ variables over the rational numbers $\mathbb{Q}$, and by $Q$ a finite quiver with vertices $1, \ldots, n$, and with no oriented cycles. Then $\underline{x}=\left\{x_{1}, \ldots, x_{n}\right\}$ is a cluster, and we consider the pair $(\underline{x}, Q)$ to be an initial seed. For each $i=1, \ldots, n$, we denote the mutation of a quiver $Q$ by $\mu_{i}(Q)=Q^{\prime}$. We also denote the mutation of a seed $(\underline{x}, Q)$ by $\mu_{i}(\underline{x}, Q)=\left(\underline{x^{\prime}}, Q^{\prime}\right)$. Here $x_{i}$ is replaced by another element $x_{i}^{*}$, to get the cluster $\underline{x}^{\prime}$, where $x_{i}^{*}$ is defined by a formula of the form $x_{i} \cdot x_{i}^{*}=m_{1}+m_{2}$, with $m_{1}$ and $m_{2}$ monomials depending on $\underline{x}$ and $Q$. The precise definitions of $Q^{\prime}$ and $x_{i}^{*}$ are given in [FZ1]; see also [BMR2]. Continuing this way we get a collection of seeds. The $n$-element subsets of the seeds are the clusters, and the elements in the clusters are the cluster variables. The associated cluster algebra is the subalgebra $\mathcal{A}(Q)$ of $\mathbb{Q}\left(x_{1}, \ldots, x_{n}\right)$ over $\mathbb{Q}$ generated by the cluster variables. We can start with any of the seeds as the initial seed. The cluster algebras we have defined here are said to be acyclic, meaning that one of the quivers occurring in some seed, in this case $Q$ itself, has no oriented cycles [BFZ]. 
1.2. Denominators of cluster variables. When expressing a cluster variable in terms of the initial cluster $\underline{x}$ as a reduced fraction

$$
f / m=f\left(x_{1}, \ldots, x_{n}\right) / m\left(x_{1}, \ldots, x_{n}\right)
$$

one has the remarkable fact that $m\left(x_{1}, \ldots, x_{n}\right)$ is a monomial. This is called the Laurent phenomenon [FZ1]. For finite type, the quiver $Q$ in the initial seed can be assumed to be a Dynkin quiver [FZ2], and it is known that when a cluster variable different from $x_{1}, \ldots, x_{n}$ is expressed in terms of the cluster variables in the initial seed $(\underline{x}, Q)$, the denominator determines the composition factors of an indecomposable $k Q$-module. This was shown in [FZ2] for a Dynkin quiver with alternating orientation, and extended to an arbitrary Dynkin quiver in [CCS2] (independently $[\mathrm{RT}]$ ). A different proof was given in [CK1] based upon [CC].

1.3. Cluster categories. For a finite dimensional hereditary $k$-algebra $H$, the cluster category $\mathcal{C}_{H}=D^{b}(H) / \tau^{-1}[1]$ was introduced in [BMRRT] in order to model essential ingredients in the theory of cluster algebras (see [CCS1] for a category associated with the Dynkin diagram $A_{n}$, shown to be equivalent to the corresponding cluster category). Here $D^{b}(H)$ denotes the bounded derived category of $H$, with suspension functor [1], and $\tau$ denotes the AR-translation on $D^{b}(H)$, induced by the corresponding translation for $H$-modules. The category $\mathcal{C}_{H}$ is known to be triangulated [Kel]. Any indecomposable object in $\mathcal{C}_{H}$ is represented by an object in $D^{b}(H)$ in the fundamental domain, that is, an indecomposable $H$-module or an object of the form $P[1]$ with $P$ an indecomposable projective $H$-module. We often identify an object in $\mathcal{C}_{H}$ with its representative in the fundamental domain in $D^{b}(H)$.

Cluster-tilting theory was investigated in [BMRRT], and the (cluster-)tilting objects $T$ are the basic objects $T$ with no self-extensions such that $T$ is maximal with this property, that is, if $\operatorname{Ext}_{\mathcal{C}_{H}}^{1}(T \oplus X, T \oplus X)=0$, then $X$ is in $\operatorname{add} T$. Any tilting object is induced by a tilting module over some hereditary algebra derived equivalent to $H$. When $T=\bar{T} \oplus M$ is a basic tilting object with $M$ indecomposable, there is a unique indecomposable object $M^{*} \nsucceq M$ such that $T^{\prime}=\bar{T} \oplus M^{*}$ is a tilting object. Moreover, $M$ and $M^{*}$ are related via exchange-triangles $M^{*} \stackrel{g}{\rightarrow} B \stackrel{f}{\rightarrow} M \rightarrow$ and $M \stackrel{t}{\rightarrow} B^{\prime} \stackrel{s}{\rightarrow} M^{*} \rightarrow$ in $\mathcal{C}_{H}$ where $B$ and $B^{\prime}$ are in add $\bar{T}$, and $f$ and $s$ are minimal right add $\bar{T}$-approximations and $g, t$ are minimal left add $\bar{T}$-approximations. Here $\left(M, M^{*}\right)$ is called an exchange pair. The indecomposable objects occurring as direct summands of the tilting objects in $\mathcal{C}_{H}$ are exactly the indecomposable exceptional objects of $\mathcal{C}_{H}$ (an object $X$ of $\mathcal{C}_{H}$ is said to be exceptional if $\operatorname{Ext}_{\mathcal{C}_{H}}^{1}(X, X)=0$ ).

1.4. Cluster-tilted algebras. The algebras $\operatorname{End}_{\mathcal{C}_{H}}(T)^{\mathrm{op}}$, where $T$ is a tilting object in $\mathcal{C}_{H}$ are called cluster-tilted algebras, and a study of these algebras was initiated in [BMR1]. In the acyclic case it is known that if $Q$ is a quiver with no oriented cycles which is part of a seed and $H=k Q$, then the quivers occurring in the seeds of the cluster algebra $\mathcal{A}(Q)$ coincide with the quivers of the cluster-tilted algebras associated with $\mathcal{C}_{H}$ [BMR2]. Also the multiplication rule $x_{i} \cdot x_{i}^{*}=m_{1}+m_{2}$ appearing in the definition of a cluster algebra [FZ1] is interpreted in [BMR2] in terms of the exchange triangles in the cluster category. The multiplication rule is independently interpreted in the case of finite type in [CCS2]. 


\section{Discussion of the PRoblem/Motivation}

We recall some results from [BMR2] which are an essential starting point, and which give motivation for our later considerations.

Start with an initial seed $(\underline{x}, Q)$, where $\underline{x}=\left\{x_{1}, \ldots, x_{n}\right\}$ and $Q$ is a finite quiver without oriented cycles with vertices $1, \ldots, n$. Let $H=k Q$, and consider the associated tilting seed $(H[1], Q)$. A tilting seed is a pair $(T, \Gamma)$ where $T$ is a tilting object in $\mathcal{C}_{H}$ and $\Gamma$ is the quiver of $\operatorname{End}_{\mathcal{C}_{H}}(T)^{\mathrm{op}}$. We can associate with the cluster variable $x_{i}$ the indecomposable exceptional object $P_{i}[1]$, where $P_{i}$ is the indecomposable projective $H$-module associated with the vertex $i$. Applying the mutation $\mu_{i}$ to $(\underline{x}, Q)$ we get $\left(\underline{x}^{\prime}, Q^{\prime}\right)$, with $\underline{x}^{\prime}$ containing a new cluster variable $x_{i}^{*}$, replacing $x_{i}$. Define $\delta_{i}$ of $(H[1], Q)$ to be $\left(T^{\prime}, Q^{\prime \prime}\right)$ where $T^{\prime}$ is obtained from $H[1]$ by replacing $M=P_{i}$ [1] with a non-isomorphic indecomposable object $M^{*}$ to get a new tilting object $T^{\prime}$. Here $Q^{\prime \prime}$ is by definition the quiver of $\operatorname{End}_{\mathcal{C}_{H}}\left(T^{\prime}\right)^{\mathrm{op}}$. We then associate $M^{*}$ with $x_{i}^{*}$. Thus the elements of the new cluster $\underline{x}^{\prime}$ are associated with the summands of the tilting object $T^{\prime}$. We also know that the quivers $Q^{\prime}$ and $Q^{\prime \prime}$ are isomorphic [BMR2]. Continuing with a sequence of corresponding mutations for the seeds and for the tilting seeds, we associate in this way an indecomposable exceptional object in $\mathcal{C}_{H}$ to each cluster variable occurring for the chosen sequence of mutations. Note that, a priori, the exceptional object associated to a cluster variable is not uniquely determined by the cluster variable itself — it depends on the sequence of mutations chosen. Clusters are taken to tilting objects, and at each stage the corresponding quivers coincide.

Recall that we want to prove that a seed is determined by its cluster. The corresponding result for tilting seeds is true by definition, since the corresponding quiver is given by the endomorphism algebra of the tilting object. So it should be useful to get a closer relationship between the seeds and the tilting seeds. In particular, we show in this paper that in the above approach, the exceptional object associated to a cluster variable does not depend on the sequence of mutations used to reach it. Then a cluster $\underline{x}^{\prime}$ determines a tilting object $T^{\prime}$ uniquely and hence the quiver $Q^{\prime}$ of the tilting seed $\left(T^{\prime}, Q^{\prime}\right)$ and therefore the quiver of the seed which has cluster $\underline{x}^{\prime}$. Note that if there is a map from the set of cluster variables in $\mathcal{A}(Q)$ to the set of indecomposable exceptional objects in $\mathcal{C}_{H}$, taking $x_{i}$ to $P_{i}[1]$, and sending clusters to tilting objects, then it is uniquely determined.

So the strategy is to get enough information on the shape of each cluster variable in order to associate to it an indecomposable exceptional object in $\mathcal{C}_{H}$, in a way compatible with the above locally defined map. We can then use this to improve the results from [BMR2] and prove the desired conjecture. This will be based upon proving two theorems, stated below, which should be of independent interest. Before we state them, we introduce some more terminology.

Let $H=k Q$ and let $S_{1}, \ldots, S_{n}$ denote the simple $H$-modules associated with the vertices $1, \ldots, n$. For $M$ in $\bmod H$ denote by $[M]$ the corresponding element in the Grothendieck group $K_{0}(H)$. For a monomial $m=m\left(x_{1}, \ldots, x_{n}\right)$, denote by $\beta(m)$ the corresponding element in $K_{0}(H)$, induced by $\beta\left(x_{i}^{n_{i}}\right)=n_{i}\left[S_{i}\right]$. For a cluster variable $u$, which is not a polynomial, we write $u=f / m$ in reduced form, where $f$ is a polynomial and $m$ is a non-constant monomial with constant term 1 . If $u$ is a polynomial and $x_{i} \mid u$ for some $x_{i}$ in $\underline{x}$, we write $u=x_{i} f$. We say that a polynomial $f=f\left(x_{1}, \ldots, x_{n}\right)$ satisfies the positivity condition if $f\left(e_{i}\right)>0$ where 
$e_{i}=(1, \ldots, 1,0,1, \ldots, 1)$ (with 0 in the $i$ th position) for $i=1, \ldots, n$. The following is a crucial observation.

Lemma 2.1. Let $u=f / m$, where $f$ is a polynomial satisfying the positivity condition and $m$ is a non-constant monomial. Then $u=f / m$ is in reduced form.

We say that a cluster variable expressed in terms of the initial seed $(\underline{x}, Q)$, where $Q$ is a quiver without oriented cycles, is of type $(\star)$ if

- It is of the form $f / m$ or $f x_{i}$ where $m$ is a non-constant monomial and $f$ is a polynomial satisfying the positivity condition.

- In case it is equal to $f / m$, there is an exceptional $H$-module $M$, such that $\beta(m)=[M]$.

Recall that an indecomposable exceptional $H$-module $M$ is uniquely determined by $[M]$ (see $[\mathrm{Ker}]$ ). Thus, the $M$ appearing in the above definition is uniquely defined.

Our first main result is the following.

Theorem 2.2. The cluster variables for the cluster algebra $\mathcal{A}(Q)$ are all of type (*). For each non-polynomial cluster variable $f / m$, there is a unique indecomposable exceptional $H$-module $M$ such that $\beta(m)=[M]$, and each indecomposable exceptional module corresponds to the denominator of some cluster variable.

We define a map $\alpha$ from cluster variables of type $(\star)$ to indecomposable objects in $\mathcal{C}_{H}$, with $H=k Q$, as follows:

(i) $\alpha(f / m)=M$, where $M$ is the indecomposable exceptional object with $\beta(m)=[M]$

(ii) $\alpha\left(f x_{i}\right)=P_{i}[1]$, where $P_{i}$ is the projective cover of $S_{i}$.

Let $c_{M}=\beta^{-1}[M]$, that is, the exponents of $c_{M}$ give the composition factors of $M$.

There is an induced map $\bar{\alpha}$ defined on the clusters consisting of cluster variables of type $(\star)$ to the set of tilting objects, and an induced map $\widetilde{\alpha}$ from seeds with the same condition on the involved clusters, to the set of tilting seeds.

The map $\alpha$ is used in the proof of Theorem 2.2, and it is clear that when this proof is finished, the map $\alpha$ is defined on the set of cluster variables, and hence $\bar{\alpha}$ and $\widetilde{\alpha}$ are defined on the sets of clusters and seeds, respectively.

Note that if $X$ is an indecomposable $H$-module, then by [BMRRT] we have

$$
\operatorname{Ext}_{\mathcal{C}_{H}}^{1}(X, X) \simeq \operatorname{Ext}_{H}^{1}(X, X) \oplus D \operatorname{Ext}_{H}^{1}(X, X),
$$

so that $X$ is an exceptional $H$-module if and only if it is an exceptional object in $\mathcal{C}_{H}$. Also $P_{i}[1]$ is an indecomposable exceptional object in $\mathcal{C}_{H}$.

The second theorem is the following.

Theorem 2.3. Let $Q$ be a finite quiver with no oriented cycles, and let $H=k Q$. The map

$\alpha:\{$ cluster variables of $\mathcal{A}(Q)\} \rightarrow\left\{\right.$ indecomposable exceptional objects in $\left.\mathcal{C}_{H}\right\}$ is surjective, it induces a surjective map $\bar{\alpha}:\{$ clusters $\} \rightarrow\{$ tilting objects $\}$, and a surjective map $\widetilde{\alpha}:\{$ seeds $\} \rightarrow\{$ tilting seeds $\}$, preserving quivers.

Those two results will actually be proven together, where the crucial induction step is treated in the next section. 


\section{The Induction STEP}

In this section we give the induction step needed for the proof of Theorems 2.2 and 2.3. As before $(\underline{x}, Q)$, with $\underline{x}=\left\{x_{1}, \ldots, x_{n}\right\}$, denotes the initial seed, with $Q$ a finite quiver with no oriented cycles and vertices $1, \ldots, n$.

Proposition 3.1. Let $\left(\underline{x}^{\prime}, Q^{\prime}\right)$ be a seed, with $\underline{x}^{\prime}=\left\{x_{1}^{\prime}, \ldots, x_{n}^{\prime}\right\}$, and choose some $x_{i}^{\prime}$. Assume that all $x_{j}^{\prime}$ are of type $(\star)$, and that $\alpha\left(x_{1}^{\prime}\right) \oplus \cdots \oplus \alpha\left(x_{n}^{\prime}\right)=T^{\prime}=M \oplus \bar{T}$ is a tilting object in $\mathcal{C}_{H}$, where $M=\alpha\left(x_{i}^{\prime}\right)$, and $Q^{\prime}$ is the quiver of $\operatorname{End}_{\mathcal{C}_{H}}\left(T^{\prime}\right)^{\mathrm{op}}$. For an indecomposable direct summand $A$ of $T^{\prime}$, denote the corresponding cluster variable in $\underline{x}^{\prime}$ by $x_{A}$. Then we have the following:

(a) Performing the mutation $\mu_{i}$ on $\left(\underline{x}^{\prime}, Q^{\prime}\right)$, the new cluster variable $\left(x_{i}^{\prime}\right)^{*}=$ $\left(x_{M}\right)^{*}$ is also of type $(*)$, and $\alpha\left(\left(x_{M}\right)^{*}\right)=M^{*}$, where $M^{*}$ is the unique indecomposable object in $\mathcal{C}_{H}$ with $M^{*} \nsucceq M$ and $\bar{T} \oplus M^{*}$ a tilting object.

(b) If $\left(\underline{x}^{\prime \prime}, Q^{\prime \prime}\right)$ is the new seed, where $\underline{x}^{\prime \prime}=\left\{x_{1}^{\prime}, \ldots,\left(x_{i}^{\prime}\right)^{*}, \ldots, x_{n}^{\prime}\right\}$, then

$$
\alpha\left(x_{1}^{\prime}\right) \oplus \cdots \oplus \alpha\left(\left(x_{i}^{\prime}\right)^{*}\right) \oplus \cdots \oplus \alpha\left(x_{n}^{\prime}\right)=\bar{T} \oplus M^{*},
$$

so that the cluster $\underline{x}^{\prime \prime}$ is taken to the tilting object $T^{\prime \prime}=\bar{T} \oplus M^{*}$, and $Q^{\prime \prime}$ is the quiver of $\operatorname{End}_{\mathcal{C}_{H}}\left(T^{\prime \prime}\right)^{\mathrm{op}}$, so that the seed $\left(\underline{x}^{\prime \prime}, Q^{\prime \prime}\right)$ is taken to the tilting seed $\left(T^{\prime \prime}, Q_{T^{\prime \prime}}\right)$, where $Q^{\prime \prime}=Q_{T^{\prime \prime}}$.

Proof. Let $B \rightarrow M$ and $M \rightarrow B^{\prime}$ be minimal right and left add $\bar{T}$-approximations of $M$, respectively. If $A$ is an indecomposable direct summand of $M \oplus B \oplus B^{\prime}$, then it is an indecomposable direct summand of $T^{\prime}$. Hence we have by assumption that the cluster variable $x_{A}$ is of type $(\star)$, that is, $x_{A}=f_{A} / m_{A}$, with $\beta\left(m_{A}\right)=A$ if $A$, chosen in the fundamental domain, is an $H$-module, and $x_{A}=f_{A} \cdot x_{i}=f_{A} \cdot c_{S_{i}}$ if $A=P_{i}$ [1] with $S_{i}$ the simple module corresponding to the vertex $i$, and $P_{i}$ the projective cover of $S_{i}$. In both cases $f_{A}$ satisfies the positivity condition.

Consider the approximation triangles

$$
M^{*} \rightarrow B \rightarrow M \rightarrow \text { and } M \rightarrow B^{\prime} \rightarrow M^{*} \rightarrow
$$

where $B=\bigoplus_{i} B_{i}$, and $B=\bigoplus B_{j}^{\prime}$, with the $B_{i}$ and $B_{j}^{\prime}$ indecomposable.

We have by [BMR2] that

$$
x_{M} \cdot\left(x_{M}\right)^{*}=x_{B}+x_{B^{\prime}},
$$

where $x_{B}=\prod x_{B_{i}}$ and $x_{B^{\prime}}=\prod x_{B_{j}^{\prime}}$. For further analysis we consider two different cases.

CASE I.

Assume that we have an exact sequence $0 \rightarrow M^{*} \rightarrow B \rightarrow M \rightarrow 0$ of $H$ modules. Since $M^{*}$ is not injective, $\tau^{-1} M^{*}$ is an $H$-module. By [BMRRT], we have that $D \operatorname{Ext}_{\mathcal{C}_{H}}^{1}\left(M, M^{*}\right) \simeq \operatorname{Hom}_{\mathcal{C}_{H}}\left(M^{*}, \tau M\right) \simeq \operatorname{Hom}_{\mathcal{C}_{H}}\left(\tau^{-1} M^{*}, M\right)$ is 1dimensional over $k$. Since $\operatorname{Hom}_{D^{b}(H)}\left(\tau^{-1} M^{*}, \tau^{-1} M[1]\right)=\operatorname{Ext}_{H}^{1}\left(M^{*}, M\right)=0$, because $\operatorname{Ext}_{H}^{1}\left(M, M^{*}\right) \neq 0$, there is a non-zero module map $h: \tau^{-1} M^{*} \rightarrow M$. Consider the corresponding exact sequence

$$
0 \rightarrow K_{h} \rightarrow \tau^{-1} M^{*} \stackrel{h}{\rightarrow} M \rightarrow L_{h} \rightarrow 0 .
$$

This gives rise to the triangle

$$
M \rightarrow L_{h} \oplus K_{h}[1] \rightarrow M^{*} \rightarrow
$$

in $D^{b}(H)$, whose image in $\mathcal{C}_{H}$ is (isomorphic to) the exchange triangle

$$
M \rightarrow B^{\prime} \rightarrow M^{*} \rightarrow \text {. }
$$


Write $K_{h}=P \oplus K_{h}^{\prime}$, where $P$ is projective and $K_{h}^{\prime}$ has no non-zero projective direct summands. Then, in the cluster category, we have $B^{\prime} \simeq L_{h} \oplus K_{h}^{\prime}[1] \simeq L_{h} \oplus \tau K_{h}^{\prime} \oplus$ $P[1]$. Here, $L_{h} \oplus \tau K_{h}^{\prime} \oplus P[1]$ is in the fundamental domain in $D^{b}(H)$. Since we have a monomorphism $K_{h} \rightarrow \tau^{-1} M^{*}$, we also have a monomorphism $\tau K_{h}^{\prime} \rightarrow M^{*}$. Letting $A=L_{h} \oplus \tau K_{h}^{\prime}$, we see that $[B]=[M]+\left[M^{*}\right]=[A]+[C]$, for some $H$-module $C$, using that $L_{h}$ is a factor of $M$, and that $\tau K_{h}^{\prime}$ is a submodule of $M^{*}$. For a direct summand $Y=Y_{1} \oplus \cdots \oplus Y_{r}$ of $T^{\prime}$, where the $Y_{j}$ are indecomposable, we write $f_{Y}=f_{Y_{1}} \cdots f_{Y_{r}}, m_{Y}=m_{Y_{1}} \cdots m_{Y_{r}}$ and $x_{Y}=x_{Y_{1}} \cdots x_{Y_{r}}$. Then we have $x_{B^{\prime}}=$ $x_{A} \cdot x_{P[1]}=\left(f_{A} / m_{A}\right) \cdot c_{S} \cdot f_{P[1]}$, where $S=P / \underline{\mathrm{r}} P$. Here $f_{A}$ satisfies the positivity condition, since $f_{A^{\prime}}$ satisfies the positivity condition for each indecomposable direct summand $A^{\prime}$ of $A$. We have $m_{B}=m_{A} \cdot m^{\prime}$, where $m^{\prime}$ is a monomial, using the fact that $m_{B}=c_{B}$ and $m_{A}=c_{A}$.

By the interpretation of the multiplication $x_{M} \cdot\left(x_{M}\right)^{*}$ in the cluster category from [BMR2], we have that

$$
x_{M} \cdot\left(x_{M}\right)^{*}=x_{B}+x_{B^{\prime}}=\frac{f_{B}}{m_{B}}+\frac{f_{A}}{m_{A}} \cdot c_{S} \cdot f_{P[1]} .
$$

so that

$$
\left(x_{M}\right)^{*}=\frac{\left(f_{B}+f_{A} \cdot c_{S} \cdot f_{P[1]} \cdot m^{\prime}\right) \cdot m_{M}}{m_{B} \cdot f_{M}} .
$$

It follows from the exact sequence $0 \rightarrow M^{*} \rightarrow B \rightarrow M \rightarrow 0$ that $m_{B} / m_{M}=c_{M^{*}}$, the monomial corresponding to the composition factors of $M^{*}$. We have

$$
\left(x_{M}\right)^{*}=\frac{\left(f_{B}+f_{A} \cdot c_{S} \cdot f_{P[1]} \cdot m^{\prime}\right) \cdot \frac{1}{f_{M}}}{c_{M^{*}}},
$$

Note that as $f_{M}$ is assumed to satisfy the positivity condition, it can have no monomial factor, and it follows from the Laurent phenomenon that the numerator is a polynomial. We want to show the desired positivity condition for the numerator, which in particular will imply that the above expression for $\left(x_{M}\right)^{*}$ is in reduced form: For $e_{i}=(1, \ldots, 1,0,1, \ldots, 1)$ (the 0 in the $i$ 'th position) we have by assumption, or directly, that $f_{B}\left(e_{i}\right)>0, f_{A}\left(e_{i}\right)>0, f_{P[1]}\left(e_{i}\right)>0, m^{\prime}\left(e_{i}\right) \geq 0, c_{S}\left(e_{i}\right) \geq 0$ and $f_{M}\left(e_{i}\right)>0$, so overall we get a positive number. The expression is thus in its reduced form, and hence we get $m_{M^{*}}=c_{M^{*}}$ and consequently $\alpha\left(\left(x_{M}\right)^{*}\right)=M^{*}$, as desired.

If $M$ and $M^{*}$ are still assumed to be $H$-modules, and there is no non-split exact sequence $0 \rightarrow M^{*} \rightarrow B \rightarrow M \rightarrow 0$ of $H$-modules, then we have a non-split exact sequence $0 \rightarrow M \rightarrow B^{\prime} \rightarrow M^{*} \rightarrow 0$ of $H$-modules, by [BMRRT]. This amounts to, using the previous notation, assuming our claims are proved for $B, B^{\prime}$ and $M^{*}$, and then showing the desired property for $M$. So the equation (1) is replaced by

$$
x_{M^{*}} \cdot\left(x_{M^{*}}\right)^{*}=\frac{f_{B}}{m_{B}}+\frac{f_{A}}{m_{A}} \cdot c_{S} \cdot f_{P[1]} .
$$

We then get

$$
\left(x_{M^{*}}\right)^{*}=\frac{\left(f_{B}+f_{A} \cdot c_{S} \cdot f_{P[1]} \cdot m^{\prime}\right) \cdot \frac{1}{f_{M^{*}}}}{c_{M}},
$$

and we similarly get that $c_{M}=m_{M}$ and $\alpha\left(\left(x_{M^{*}}\right)^{*}\right)=M$. 
CASE II.

Assume now that $M$ is not induced by a module, hence $M=P[1]$ for an indecomposable projective $H$-module $P$. Since $\operatorname{Ext}_{\mathcal{C}_{H}}^{1}\left(M, M^{*}\right) \neq 0$, we have that $M^{*}$ cannot be of the form $Q[1]$, with $Q$ projective, and is hence an $H$-module.

Consider the diagram

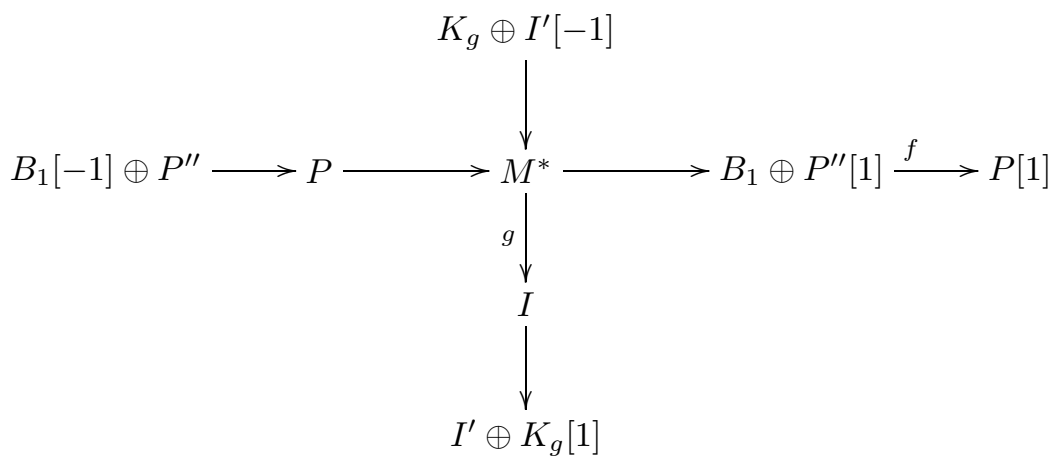

where $f$ is a minimal right add $\bar{T}$-approximation of $M=P[1]$, and $B=B_{1} \oplus P^{\prime \prime}[1]$, where $B_{1}$ is an $H$-module and $P^{\prime \prime}$ a projective $H$-module, $I=\tau(P[1])$ is the indecomposable injective $H$-module with socle isomorphic to the simple module $S=P / \underline{\mathrm{r}} P$, and $g: M^{*} \rightarrow I$ a non-zero map in the cluster category, and hence in the module category, since $I$ is injective. We have that $\operatorname{Hom}_{\mathcal{C}}\left(M^{*}, I\right) \simeq \operatorname{Hom}_{H}\left(M^{*}, I\right)$ is 1-dimensional, since $\operatorname{Hom}_{\mathcal{C}}\left(M^{*}, I\right)=\operatorname{Hom}_{\mathcal{C}}\left(M^{*}, \tau P[1]\right)=\operatorname{Hom}_{\mathcal{C}}\left(M^{*}, \tau M\right)=$ $\operatorname{Hom}_{\mathcal{C}}\left(M^{*}, M[1]\right)$ and $\operatorname{Hom}_{\mathcal{C}}\left(M^{*}, M[1]\right)$ is 1-dimensional. We have in addition that $I[-1] \simeq P[1]$ in the cluster category. Hence it is clear that the image of the triangle induced from the map $g$ is indeed isomorphic to the exchange triangle $M \rightarrow$ $B^{\prime} \rightarrow M^{*} \rightarrow$. This means that we have the minimal right add $\bar{T}$-approximation $B^{\prime}=K_{g} \oplus I^{\prime}[-1] \rightarrow M^{*}$ of $M^{*}$, where $I^{\prime}=$ Coker $g$ and $K_{g}=\operatorname{Ker} g$. Here $I^{\prime}$ is an injective $H$-module, being a factor of the injective $H$-module $I$. Denote its socle by $S^{\prime}$, and let $P^{\prime}$ be the projective cover of $S^{\prime}$. We have exact sequences $0 \rightarrow P^{\prime \prime} \rightarrow P \rightarrow M^{*} \rightarrow B_{1} \rightarrow 0$ and $0 \rightarrow K_{g} \rightarrow M^{*} \rightarrow I \rightarrow I^{\prime} \rightarrow 0$. Consider the exact commutative diagram

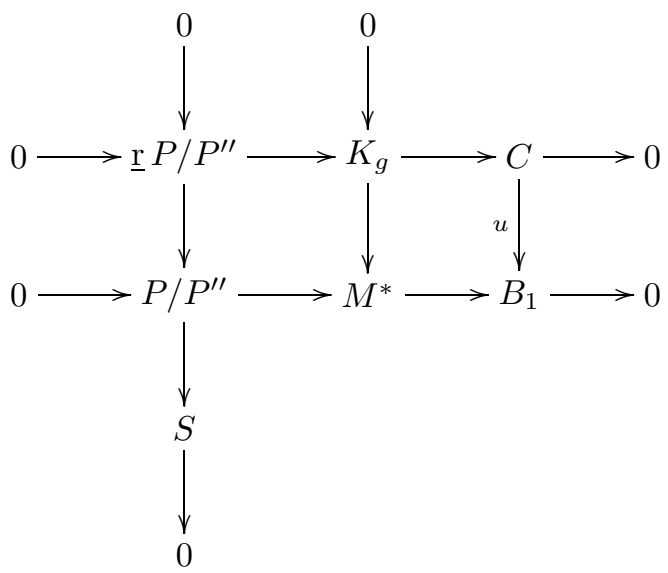

Here we use that $\underline{\mathrm{r}} P / P^{\prime \prime} \subseteq K_{g}$, since the composition $P \rightarrow M^{*} \rightarrow I$ has image in the simple socle $S$ of $I$. Since $\operatorname{dim}_{k} \operatorname{Hom}\left(M^{*}, I\right)=1$, we have that $S$ occurs exactly 
once as a composition factor of $M^{*}$. It also occurs exactly once as a composition factor of $I$ as there are no loops in the quiver of $H$. The exact sequence $0 \rightarrow K_{g} \rightarrow$ $M^{*} \rightarrow I$ then shows that $S$ is not a composition factor of $K_{g}$, and hence not of $C$ or of Ker $u$. By the snake lemma applied to the above diagram we have an exact sequence $0 \rightarrow \operatorname{Ker} u \rightarrow S$, which shows that $u: C \rightarrow B_{1}$ is a monomorphism.

We have by assumption that the cluster variables in $\underline{x^{\prime}}$ corresponding to indecomposable direct summands of $B, B^{\prime}$ and $M$ are of type $(\star)$. Hence we have $x_{P[1]}=f_{P[1]} \cdot x_{S}$ and $x_{P^{\prime \prime}[1]}=f_{P^{\prime \prime}[1]} \cdot x_{S^{\prime \prime}}$ and $x_{P^{\prime}[1]}=f_{P^{\prime}[1]} \cdot x_{S^{\prime}}$, with $f_{P[1]}, f_{P^{\prime}[1]}$ and $f_{P^{\prime \prime}[1]}$ satisfying the positivity condition, and $S^{\prime \prime}=P^{\prime \prime} / \underline{\mathrm{r}} P^{\prime \prime}$. We also have $m_{K_{g}}=c_{K_{g}}$ and $m_{B_{1}}=c_{B_{1}}$. This gives

$$
\begin{aligned}
x_{M} \cdot\left(x_{M}\right)^{*} & =x_{B}+x_{B^{\prime}} \\
& =x_{B_{1}} \cdot x_{P^{\prime \prime}[1]}+x_{K_{g}} \cdot x_{P^{\prime}[1]} \\
& =\frac{f_{B_{1}}}{c_{B_{1}}} \cdot c_{S^{\prime \prime}} \cdot f_{P^{\prime \prime}[1]}+\frac{f_{K_{g}}}{c_{K_{g}}} \cdot c_{S^{\prime}} \cdot f_{P^{\prime}[1]} .
\end{aligned}
$$

By the above exact sequences we have $c_{B_{1}}=c_{B_{1} / C} \cdot c_{C}$ and $c_{K_{g}}=c_{\underline{\mathrm{r}} P / P^{\prime \prime}} \cdot c_{C}$ and $\left[M^{*}\right]=\left[P / P^{\prime \prime}\right]+\left[B_{1}\right]=\left[\underline{\mathrm{r}} P / P^{\prime \prime}\right]+[S]+\left[B_{1}\right]$, so that $c_{M^{*}} / c_{S}=c_{\underline{\mathrm{r}} P / P^{\prime \prime}} \cdot c_{B_{1} / C} \cdot c_{C}$. This gives, using $x_{M}=x_{P[1]}=f_{P[1]} \cdot x_{S}$, that

$$
\left(x_{M}\right)^{*}=\frac{\left(c_{\underline{r} P / P^{\prime \prime}} \cdot f_{B_{1}} \cdot f_{P^{\prime \prime}[1]} \cdot c_{S^{\prime \prime}}+c_{B_{1} / C} \cdot f_{K_{g}} \cdot f_{P^{\prime}[1]} \cdot c_{S^{\prime}}\right) \cdot \frac{1}{f_{M}}}{c_{M^{*}}} .
$$

We want to show that $\underline{\mathrm{r}} P / P^{\prime \prime} \oplus S^{\prime \prime}$ and $B_{1} / C \oplus S^{\prime}$ have no common composition factors, so that no $x_{i}$ divides $c_{\underline{\mathrm{r}} P / P^{\prime \prime}} \cdot c_{S^{\prime \prime}}$ and $c_{B_{1} / C} \cdot c_{S^{\prime}}$. If $S_{1}$ is a simple $H$ module which is a composition factor of $\underline{\mathrm{r}} P / P^{\prime \prime} \oplus S^{\prime \prime}$, there must be a path in the quiver of $H$ from the vertex corresponding to $S$ to the vertex corresponding to $S_{1}$. We have $M^{*} / K_{g} \subseteq I$, and $I^{\prime}$ is a factor of $I$. If $S_{1}$ is a composition factor of $B_{1} / C \oplus S^{\prime}$, and hence of $M^{*} / K_{g} \oplus S^{\prime}$, we would have a path from the vertex corresponding to $S_{1}$ to the vertex corresponding to $S$. Since the quiver of $H$ has no oriented cycles, we conclude that no $x_{i}$ divides both $c_{\underline{\mathrm{r}} P / P^{\prime \prime}} \cdot c_{S^{\prime \prime}}$ and $c_{B_{1} / C} \cdot c_{S^{\prime}}$. Since $f_{B_{1}}, f_{K_{g}}, f_{M}, f_{P^{\prime \prime}[1]}$ and $f_{P^{\prime}[1]}$ satisfy the positivity condition, it follows that the numerator in the above expression for $\left(x_{M}\right)^{*}$ also does. Since the denominator gives the composition factors for the indecomposable exceptional $H$-module $M^{*}$, the cluster variable $\left(x_{M}\right)^{*}$ is of the desired form, and we have $\alpha\left(\left(x_{M}\right)^{*}\right)=M^{*}$.

The case where $M^{*}=P[1]$ and $M$ is an $H$-module follows by symmetry as in Case I. In view of the discussions in Section 2, this finishes the proof. We have then proved (a). The first part of (b) then follows directly, and it follows from [BMR2], as discussed in Section 2, that $\left(\underline{x}^{\prime \prime}, Q^{\prime \prime}\right)$ and $\tilde{\alpha}\left(\underline{x}^{\prime \prime}, Q^{\prime \prime}\right)$ have the same quiver.

\section{Proof of main Result}

In this section we apply the induction step to give the proof of Theorems 2.2 and 2.3, and we give our application to seeds being determined by clusters.

Proof of Theorems 2.2 and 2.3. Let $(\underline{x}, Q)$, with $\underline{x}=\left\{x_{1}, \ldots, x_{n}\right\}$, be an initial seed, where $Q$ has no oriented cycles. Then $x_{1}, \ldots, x_{n}$ are clearly all of the desired form, and $\alpha\left(x_{i}\right)$ is defined to be $P_{i}[1]$. Then $\alpha\left(x_{1}\right) \oplus \cdots \oplus \alpha\left(x_{n}\right)=P_{1}[1] \oplus \cdots \oplus P_{n}[1]$ is a tilting object in $\mathcal{C}_{H}$, with $H=k Q$, so the seed $(\underline{x}, Q)$ corresponds to the tilting seed $(H[1], Q)$, which is the start of our induction.

Consider the following property $(\widetilde{\star})$ of a seed $(\underline{u}, \Gamma)$, where $\underline{u}=\left\{u_{1}, \ldots, u_{n}\right\}$. 
Property $(\widetilde{)})$. The cluster variables $u_{1}, \ldots, u_{n}$ are of type $(\star)$, so that $\alpha\left(u_{i}\right)$ is defined for $i=1, \ldots, n$, the induced map $\bar{\alpha}$ takes the cluster $\underline{u}$ to the tilting object $T=\alpha\left(u_{1}\right) \oplus \cdots \oplus \alpha\left(u_{n}\right)$, and the induced map $\widetilde{\alpha}$ takes the seed $(\underline{u}, \Gamma)$ to the tilting seed $\left(T, Q_{T}\right)$, where $\Gamma=Q_{T}$.

By the above remarks, the initial seed $(\underline{x}, Q)$ satisfies $(\widetilde{\star})$. Let $\left(\underline{x}^{\prime \prime}, Q^{\prime \prime}\right)$ be a seed where $t>0$ is the number of mutations in a minimal sequence of mutations from $(\underline{x}, Q)$ to $\left(\underline{x}^{\prime \prime}, Q^{\prime \prime}\right)$. Assume that all seeds reached by a sequence of mutations in a minimal sequence of mutations of length less than $t$ have property $(\widetilde{\star})$. Then choose $\left(\underline{x}^{\prime}, Q^{\prime}\right)$, such that $\left(\underline{x}^{\prime \prime}, Q^{\prime \prime}\right)=\mu_{i}\left(\underline{x}^{\prime}, Q^{\prime}\right)$ for some $i$, and $\left(\underline{x}^{\prime}, Q^{\prime}\right)$ is reached by a minimal sequence of mutations of length $t-1$. Then it follows directly from Proposition 3.1 that $\left(\underline{x}^{\prime \prime}, Q^{\prime \prime}\right)$ satisfies $(\widetilde{\star})$.

It then only remains to show that $\alpha, \bar{\alpha}$ and $\widetilde{\alpha}$ are surjective. Let $T_{i}^{\prime}$ be an exceptional object in $\mathcal{C}_{H}$. It is then a direct summand of a tilting object $T^{\prime}$. Since the cluster-tilting graph is connected (see [BMRRT]), the corresponding tilting seed $\left(T^{\prime}, Q^{\prime}\right)$ is obtained from $(H[1], Q)$ by a finite number of mutations of tilting seeds. We do the corresponding sequence of mutations of seeds to the initial seed $(\underline{x}, Q)$. Then we are in the above situation, and it follows that all the maps $\alpha, \bar{\alpha}$ and $\widetilde{\alpha}$ are surjective.

The following was conjectured by Fomin and Zelevinsky [FZ2] (for any cluster algebra).

Theorem 4.1. For an acyclic cluster algebra (associated with a skew-symmetric matrix and with no coefficients) a seed is determined by its cluster.

Proof. We can choose an initial cluster $(\underline{x}, Q)$, where $Q$ is a quiver with no oriented cycles, and express the cluster variables in terms of the variables $x_{1}, \ldots, x_{n}$ in $\underline{x}$. Let $\underline{x}^{\prime}$ be a cluster, and choose a seed $\left(\underline{x}^{\prime}, Q^{\prime}\right)$. Consider the tilting seed $\widetilde{\alpha}\left(\underline{x}^{\prime}, Q^{\prime}\right)=$ $\left(\bar{\alpha}\left(\underline{x}^{\prime}\right), Q^{\prime}\right)$. Since $\bar{\alpha}\left(\underline{x}^{\prime}\right)$ determines $Q^{\prime}$, it follows that the cluster $\underline{x}^{\prime}$ determines the seed $\left(\underline{x}^{\prime}, Q^{\prime}\right)$.

\section{Concluding Remarks}

Note that for finite type it is known [FZ2] that the number of cluster variables is equal to the number of almost positive roots, and hence to the number of indecomposable objects (which are all exceptional) in the associated cluster category. Hence knowing that $\alpha$ is surjective, we can conclude from this that $\alpha$ is actually a bijection. As a consequence, this gives an alternative proof of the correspondence theorem, taking clusters to tilting objects, in [BMRRT].

If one is only interested in the shape of the denominator for cluster variables for finite type, one can get a more direct proof, still using our notion of positivity: define a map $\psi$ : ind $\mathcal{C}_{H} \rightarrow$ \{cluster variables $\}$ by sending $P_{i}[1]=I_{i}[-1]$ to $x_{i}$ as usual, and using the almost split triangles corresponding to the exchange pairs $\left(I_{i}[-1], P_{i}\right)$ to get cluster variables of the desired form corresponding to the indecomposable projectives. Then use almost split sequences to get cluster variables of the desired form corresponding to all the other objects. Then $\psi$ will clearly be $1-1$, and hence bijective by the above mentioned result from [FZ2].

When $H$ is an indecomposable hereditary $k$-algebra of infinite type, we can use the same procedure to get a 1-1 map $\psi$ from the indecomposable preprojective $H$ modules to the cluster variables. This gives in our setting a short proof for the fact 
that if the quiver of $H$ is not Dynkin, the associated cluster algebra is of infinite type, which is part of [FZ2, Th. 1.5].

\section{Appendix A: Clusters and tilting objects \\ FOR ACYCLIC CLUSTER ALGEBRAS}

By Aslak Bakke Buan, Philippe Caldero, Bernhard Keller,

Bethany R. Marsh, Idun Reiten, and Gordana Todorov

Let $H$ be a finite dimensional hereditary algebra over an algebraically closed field $k$ with associated cluster category $\mathcal{C}_{H}$ and cluster algebra $\mathcal{A}_{H}$. Let ind exc $\mathcal{C}_{H}$ denote the set of isomorphism classes of indecomposable exceptional objects in $\mathcal{C}_{H}$. The purpose of this appendix is to point out that combining the surjective Caldero-Chapoton map $\beta$ from [CK2], with the surjective map $\alpha$ in this paper gives the following stronger result, confirming Conjecture 9.1 in [BMRRT], as a direct consequence. It gives a refinement of Theorem 4 in [CK2], which is proved using the surjective map $\beta$, together with the idea of positivity from this paper.

Theorem A.1. The surjective maps

$$
\alpha:\{\text { cluster variables }\} \rightarrow\left\{\text { ind } \operatorname{exc} \mathcal{C}_{H}\right\}
$$

and

$$
\beta:\left\{\text { ind exc } \mathcal{C}_{H}\right\} \rightarrow\{\text { cluster variables }\}
$$

are inverse bijections, inducing inverse bijections between clusters and tilting objects and between seeds and tilting seeds.

Proof. Consider the surjective map

$$
\alpha \beta:\left\{\text { ind exc } \mathcal{C}_{H}\right\} \rightarrow\left\{\text { ind } \operatorname{exc} \mathcal{C}_{H}\right\} .
$$

It is clear from the definition of $\alpha$ and $\beta$, that $\alpha \beta$ is the identity on the indecomposable direct summands of $H[1]$. It follows from Theorem 2.3 and the part of Theorem 4 in [CK2] that does not depend on this paper, that $\alpha \beta$ takes tilting objects to tilting objects and tilting seeds to tilting seeds. It then follows from the recurrence relations satisfied by the maps that $\alpha \beta$ is the identity, and hence $\alpha$ and $\beta$ are inverse bijections.

The following improvement of Theorem 2.2 follows directly.

Corollary A.2. The maps $\alpha$ and $\beta$ give inverse $1-1$ correspondences between the non-polynomial cluster variables and the isomorphism classes of indecomposable $H$ modules, such that the denominator of a cluster variable is given by the composition factors of the corresponding indecomposable $H$-module.

Another direct consequence is the following generalization of a result proved for finite type in [FZ2].

Corollary A.3. Let $\underline{u}=\left\{u_{1}, u_{2}, \ldots, u_{n}\right\}$ be a cluster for $\mathcal{A}_{H}$. Then for each $i=1, \ldots, n$ there is exactly one cluster variable $u_{i}^{*} \neq u_{i}$ such that

$$
\left\{u_{1}, \ldots, u_{i-1}, u_{i}^{*}, u_{i+1}, \ldots, u_{n}\right\}
$$

is a cluster. 


\section{REFERENCES}

[BFZ] Berenstein A., Fomin S., Zelevinsky A. Cluster Algebras III: Upper bounds and double Bruhat cells. Duke Math J. 126 (2005), no. 1, 1-52. MR2110627 (2005i:16065)

[BMR1] Buan A., Marsh B., Reiten I. Cluster-tilted algebras, Trans. Amer. Math. Soc., 359 (2007), no. 1, 323-332. MR2247893 (2007f:16035)

[BMR2] Buan A., Marsh B., Reiten I. Cluster mutation via quiver representations, preprint arxiv:math.RT/0412077, to appear in Commentarii Mathematici Helvetici.

[BMRRT] Buan A., Marsh B., Reineke M., Reiten I., Todorov G. Tilting theory and cluster combinatorics, Advances in Mathematics, 204 (2), (2006), 572-618.

[CC] Caldero P., Chapoton F. Cluster algebras as Hall algebras of quiver representations, Commentarii Mathematici Helvetici, 81, (2006), 595-616.

[CCS1] Caldero P., Chapoton F., Schiffler R., Quivers with relations arising from clusters $\left(A_{n}\right.$ case), Trans. Amer. Math. Soc. 358 (2006), 1347-1364. MR2187656

[CCS2] Caldero P., Chapoton F., Schiffler R., Quivers with relations and cluster tilted algebras. Algebras and Representation Theory, 9, (2006), no. 4, 359-376.

[CK1] Caldero P., Keller B. From triangulated categories to cluster algebras, to appear in Inventiones Math.

[CK2] Caldero P., Keller B. From triangulated categories to cluster algebras II, Annales Scientifiques de l'Ecole Normale Supérieure, 4eme serie, 39, (2006), 983-1009.

[FZ1] Fomin S., Zelevinsky A. Cluster Algebras I: Foundations, J. Amer. Math. Soc. 15 (2002), no. 2, 497-529. MR1887642 (2003f:16050)

[FZ2] Fomin S., Zelevinsky A. Cluster Algebras II: Finite type classification. Invent. Math. 154 (2003), no. 1, 63-121. MR2004457 (2004m:17011)

[Kel] Keller B. On triangulated orbit categories, Doc. Math. 10 (2005), 551-581. MR2184464

[Ker] Kerner O. Representations of wild quivers, Proc. of the Workshop on Representation theory of Algebras and related topics, UNAM, Mexico, 1994 CMS Conf. Proc. 19, 65-107 (1996). MR1388560 (97e:16028)

[MRZ] Marsh B., Reineke M., Zelevinsky A., Generalized associahedra via quiver representations, Trans. Amer. Math. Soc. 355 (2003), no. 10, 4171-4186. MR1990581 (2004g:52014)

[RT] Reiten I., Todorov G. unpublished.

Institutt For MATEMATiske fag, Norges teknisk-NATURVitenskapelige Universitet, N7491 Trondheim, Norway

E-mail address: aslakb@math.ntnu.no

Department of Mathematics, University of Leicester, University Road, Leicester LE1 7RH, ENGLAND

InStitutt FOR MATEMATISKE FAG, NoRges TEKNISK-NATURVITENSKAPELIGE UNIVERSitet, N7491 Trondheim, Norway

E-mail address: idunr@math.ntnu.no

Department of Mathematics, Northeastern University, 360 Huntington Avenue, BosTON, Massachusetts 02115

E-mail address: todorov@neu.edu 